\title{
Efficacy of Papacarie for Caries Removal: An in vivo Study
}

\author{
${ }^{1}$ Sapna Konde, ${ }^{2}$ Pallavi Urs, ${ }^{3}$ Sunil Raj \\ ${ }^{1}$ Professor and Head, Department of Pedodontics and Preventive Dentistry, AECS Maaruti College of Dental Sciences \\ and Research Center, Bengaluru, Karnataka, India \\ ${ }^{2}$ P ostgraduate Student, Department of Pedodontics and Preventive Dentistry, AECS Maaruti College of Dental Sciences \\ and Research Center, Bengaluru, Karnataka, India \\ ${ }^{3}$ Professor, Department of Pedodontics and Preventive Dentistry, AECS Maaruti College of Dental Sciences \\ and Research Center, Bengaluru, Karnataka, India
}

Corres pondence: Sapna Konde, P rofessor and Head, DepartmentofP edodontics and P reventive Dentistry, AECS Maaruti College of Dental Sciences and Research Center, Bengaluru, Karnataka, India, e-mail: drsapna_konde@ yahoo.in

\section{ABSTRACT}

Objectives: Conventional caries removal is associated with unpleasant perception of drilling, requirement of local anesthesia, pressure effects on the pulp, etc. The chemomechanical method of caries removal has overcome these shortcomings. It is not only more comfortable to the patient but also preserves the healthy tissue. The present study was undertaken to evaluate and compare the time taken for caries removal, perception of pain and presence of secondary caries after a period of 10 months with Papacarie (Formulae One, Brazil 2003) and the conventional method. Bilateral primary molars in 52 children age ranging from 5-12 years were selected and divided into group I (conventional method) and group II (chemomechanical method) and compared.

Results: Mean time taken in the conventional method (14.5 $\pm 5.367 \mathrm{sec})$ was significantly higher than papain gel (11.2 $\pm 5.959 \mathrm{secs})$. The pain score in group 1 was significantly higher than in group 2. The pain experienced in the conventional method ranged from a minimum of 0 (none) to a maximum of 4 (uncomfortable). Whereas in papain gel, it ranged from 0 (none) to 2 (annoying). The presence of secondary caries after a follow-up of 10 months was not statistically significant.

Conclusion: Papacarie method seems to be promising as an alternative treatment procedure in pediatric dentistry.

Keywords: Chemomechanical, Papain, Pain.

\section{INTRODUCTION}

Restorative dentistry in children is usually considered traumatic and hard to perform in view of factors such as anxiety and expectation both by children and their parents. The main feature for aversion is the noise of the rotary instrument and anesthesia. Over the years, the carious tissue removal techniques are improving, aiming at facilitating the clinical practice. Several researchers are seeking replacement of the traditional mechanical removal of the carious tissue by alternative methods, such as the chemomechanical method of caries removal. ${ }^{1}$

In B razil, since 2003, a new formulation called Papacarie (Formula one Brazil) was introduced. It contains papain, chloramines, toluidine blue. Papain is a proteolytic enzyme. It has bactericidal, bacteriostatic and anti-inflammatory characteristics. Papain comes from the latex of the leaves and fruits of the green adult papaya, similar to the human pepsin, it acts as a debridant and anti-inflammatory agent which does not damage the healthy tissue and accelerates the cicatricial process. $^{2}$

In order to support the clinical use of this biomaterial, the present study aims at clinical evaluation and comparison of chemicomechanical method (Papacarie) and the conventional method of caries removal in primary teeth in relation to the time taken, perception of pain and presence of secondary caries after a period of 10 months.

\section{MATERIALS AND METHODS}

The present study was carried out in the Department of Pedodontics and Preventive dentistry at A ECS M aaruti Dental College and Research Center, B engaluru, K arnataka, India. Ethical clearancewas obtained from theinstitutional review board as well as written consent was obtained from parents/guardians. Bilateral primary molarsin 52 children ( 25 males and 27 femal es), age ranging from 5 to 12 years were selected by stratified random sampling method using theinclusion and exclusion criteria. The study design, objectives, potential benefits and methodology were explained to the selected children and their parents.

\section{Inclusion Criteria}

\section{Clinical Criteria}

1. Healthy cooperative children, between 5 and 12 years

2. Children having a minimum of two carious primary molars of comparable depth, not involving the pulp

3. No history of pain associated with the teeth

4. Only children whose parental consent was obtained prior to the procedure.

\section{Radiographic Criteria}

1. Carious lesion involving only dentin with no pulpal involvement

2. A bsence of periapical or interradicular pathology

3. A bsence of internal resorption of roots. 


\section{Exclusion Criteria}

1. Presence of intraoral/extraoral swellings

2. Deep dental caries involving the pulp

3. Presence of swelling/fistula in relation to the carious tooth.

\section{CARIES REMOVAL PROCEDURE}

Thorough oral prophylaxis of both upper and lower arches was done followed by initial radiographic evaluation of the target teeth. Radiographs were taken to confirm the depth of the lesion. The study sample was divided into group 1 and 2, having 52 teeth in each group.

\section{Group 1}

Caries was removed with the airotor handpiece using a straight fissure bur SF-41. A fter the completion of caries removal, an explorer was used for evaluation of the cavity. The caries removal was considered to be complete when the explorer did not get caught in the dentin, and when there was no "tug-back" sensation.

Once the cavity was confirmed free of caries, it was restored with glass ionomer cement after thorough isolation. The time taken for the procedure from the start of preparation of the cavity to the placement of the restoration was recorded with the help of a stopwatch.The patient's perception of pain was also recorded on the visual analog pain scale. Each of the grades associated with color was explained to the child.

\section{Group 2}

Caries removal was done according to the manufacturer's instructions (F ormula and acao). The carious cavity was first filled with Papacarie ${ }^{\circledR}$ gel and it was allow ed to act for 40 to 60 seconds. The softened decayed dentine was removed with the help of a spoon excavator. The complete removal of caries was confirmed using the visual and tactile method. The color of the papain gel was light blue at the beginning of application and bubbles appeared on the surface which indicated the process of degradation, and finally the gel changed to a darker shade indicating complete degradation. The color of the papain gel did not change when caries was completely removed (Fig. 1). The vitreous aspect of the cavity appeared when the cavity was free from caries. Reapplication of the gel was done if necessary, with no necessity to rinse the cavity between applications. Once the cavity was confirmed free of caries, the gel was removed by a wet cotton pellet and the patient was instructed to thoroughly rinse the mouth in water. The cavity was well isolated with cotton rolls and then restored with glass ionomer cement. The time taken for the procedure from the start of application of the papain up to the placement of the restoration was recorded in the assessment proforma.The patient's perception of pain for the procedure was recorded on the visual anal og pain scale.

The children were instructed to report to the department in case of fracture of the marginal ridge, partial or complete loss of the restoration or postoperative sensitivity. The children were

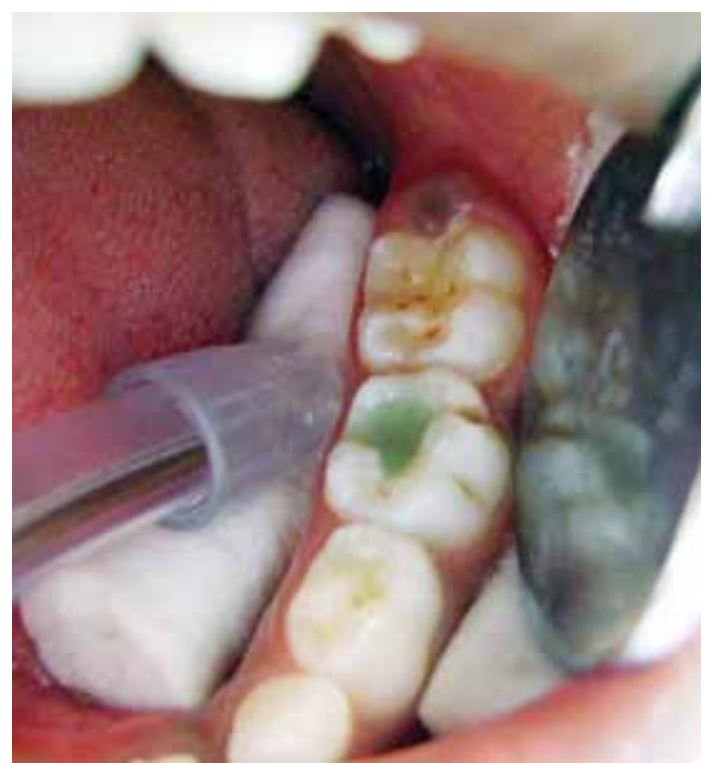

Fig. 1: Absence of color change on reapplication of the gel indicating complete caries removal by the chemomechanical method

evaluated postoperatively after a period of 10 months for secondary caries. The restoration was assessed clinically by the operator for the presence of marginal discoloration indicative of secondary caries. This was reconfirmed by an independent evaluator.

\section{RESULTS}

The results of the following study were tabulated and analyzed under the following headings:

Table 1 shows that the number of teeth treated in both group 1 (conventional method) and group 2 (papain gel) was 52. M ean time taken in group 1 was $14.5 \pm 5.367$ seconds and group 2 was $11.2 \pm 5.959$ seconds respectively. Time taken by group 2 (papain gel) was significantly less than group 1.

Table 2 shows comparison of pain score between the group 1 (conventional method) and the group 2 (papain gel method) analyzed from the visual analog pain scale cards. The mean pain in group 1 was $1.73 \pm 0.992$ and in group 2 was $0.50 \pm$ 0.610 . Subjects in group 2 showed lesser pain score than group 1 subjects and the difference was statistically significant.

Table 3 shows the distribution of pain score in the study groups. The pain experienced in the conventional method was graded from a minimum of 0 (none) to a maximum of 4 (uncomfortable). Pain score was 0 to 1 in $49(94.3 \%$ ) samples when treated with papain gel whereas $24(46.2 \%)$ when treated with conventional method. The difference observed was statistically significant.

Table 4 shows distribution of secondary caries in the study groups. The number of cases in group 1 (conventional method) that showed the presence of secondary caries after a follow-up of 10 months were eight cases (15.7\%), whereas in group 2 (papain gel) it was 11 cases (21.6\%). There was no statistical significance between the two groups. Only one tooth which was treated with papain gel had exfoliated. 
Table 1: Comparison of mean time taken between the study groups

$\begin{array}{lllllllll}\text { Group } & \text { N } & \text { Mean } & \begin{array}{l}\text { SD } \\ \text { time } \\ \text { taken }\end{array} & \text { Min. } & \text { Max. t-value p-value } \\ \text { Group 1 (C) } & 52 & 14.48 & 5.367 & 7 & 32 & 8.950 & 0.003 \\ \text { Group 2 (P) } & 52 & 11.15 & 5.959 & 5 & 45 & & \end{array}$

Table 2: Comparison of pain score between the study groups

\begin{tabular}{llllllll}
\hline Group & N & $\begin{array}{l}\text { Mean } \\
\text { pain } \\
\text { score }\end{array}$ & SD & Min. Max. t-value & p-value \\
\hline Group 1 (C) & 52 & 1.73 & 0.992 & 0 & 4 & 58.027 & $<0.001$ \\
Group 2 (P) & 52 & 0.50 & 0.610 & 0 & 2 & &
\end{tabular}

Table 3: Distribution of pain score in study groups

\begin{tabular}{lcccccc} 
Group & \multicolumn{7}{c}{ Pain score } & & & Total \\
Group 1 (C) & 0 & 1 & 2 & 3 & 4 & \\
& 3 & 21 & 19 & 5 & 4 & 52 \\
Group 2 (P) & $5.8 \%$ & $40.4 \%$ & $36.5 \%$ & $9.6 \%$ & $7.7 \%$ & $100.0 \%$ \\
& $55.8 \%$ & $38.5 \%$ & $5.8 \%$ & $0.0 \%$ & $0.0 \%$ & $100.0 \%$ \\
Total & 32 & 41 & 22 & 5 & 4 & 104 \\
& $30.8 \%$ & $39.4 \%$ & $21.2 \%$ & $4.8 \%$ & $3.8 \%$ & $100.0 \%$
\end{tabular}

Table 4: Distribution of secondary caries in study groups

\begin{tabular}{lllll}
\multirow{2}{*}{ Group } & \multicolumn{3}{c}{ Secondary caries } & \multirow{2}{*}{ Total } \\
\cline { 2 - 4 } & Absent & Present & Exfoliated & \\
Group 1 (C) & 44 & 8 & 0 & 52 \\
& $84.3 \%$ & $15.7 \%$ & $0.0 \%$ & $100.0 \%$ \\
Group 2 (P) & 40 & 11 & 1 & 52 \\
& $76.5 \%$ & $21.6 \%$ & $2.0 \%$ & $100.0 \%$ \\
Total & 84 & 19 & 1 & 104 \\
& $80.4 \%$ & $18.6 \%$ & $1.0 \%$ & $100.0 \%$
\end{tabular}

\section{DISCUSSION}

The knowledge of the carious process and the evolution of newer dental materials have led to changes in how we address and treat dental decay. The increasing need for conservative dentistry continuously gives rise to a series of trials searching for new techniques that would simplify treatment procedure. Hence, the present study evaluated the advantages of the new chemomechanical caries removal method.

The visual and tactile methods were used to eval uate caries removal in the present study. All the cavities were found to be caries-free clinically, in both conventional and Papacarie ${ }^{\circledR}$ method. ${ }^{2}$ Dyes were not used as they deeply penetrate and stain carious infected dentin as well as the porous affected dentin; hence, it may lead to over preparation of the cavity especially in primary teeth because primary dentin is more porous. ${ }^{4}$

The time taken with the initial few cases in the present study was much higher than the conventional method. However, with time and development of expertise the reverse took place with papain gel being less time consuming procedure than the conventional method. The lack of any specialized equipment and ease of procedure in the use of papain gel makes it a less time consuming procedure when compared to the conventional method and the other chemomechanical methods. W hile K otb et al reported that Papacarie was as efficient as the drill in caries removal with no significant difference in the operating time. ${ }^{4}$

However, the working time with the earlier Carisolv system was longer because of the little experience and training of operators as it had elaborated equipment. ${ }^{3}$

Self-report is the gold standard in the assessment of pain intensity. The measurement of pain intensity requires a child to choose on a rating scale, a level that best matches the amount of pain that he or she is experiencing.

The visual analog scale with color gradients provides gradations in color and area as well as length, so it is easy to see how different scale positions would reflect different levels in pain intensity. These scales have been extensively researched and they show good sensitivity and validity in children. ${ }^{5} \mathrm{H}$ ence, this scale was chosen to evaluate the patient's perception of pain in the present study.

Pain was significantly less when treated with papain gel than the conventional method. This is in accordance to a study which was evaluated by recording the pulse and blood pressure. The study indicated that in traditional drilling group, dental fear was significantly higher than that in chemomechanical and ART groups. ${ }^{6}$

Fuji IX belongs to the group of glass-ionomers with improved mechanical qualities which enable the use of the material in the posterior region. It can be used for the restoration of small lesions which are not under high occlusal load and is considered the material of choice for the modern minimal design cavities. Glass-ionomer cements designed for permanent restorations of the posterior region appear to be a material of choice for this type of minimally invasive dental restoration. ${ }^{7}$

The teeth treated with papacarie were caries-free and were clinically and radiographicaly asymptomatic with good retention of restorations after one year, indicating reduction in occurrence of secondary caries. ${ }^{8}$

Kirzioglu et al compared the chemomechanical and mechanical method of caries removal; at 3, 6, 9 and 12 months and showed that at the end of 1 year, in terms of marginal adaptation and secondary caries, there was no statistical significance which was in accordance to the present study. ${ }^{9} \mathrm{~A}$ fter a period of 10 months secondary caries in teeth treated with papain gel was $21.6 \%$ and in conventional method it was $15.7 \%$. The difference was not statistically significant. Studies of longer duration utilizing papain gel are, however, needed to confirm these findings.

\section{CONCLUSIONS}

1. Time taken for treating teeth with papacarie was significantly less as compared to the conventional method

2. Patient acceptability with papacarie was significantly higher

3. The follow-up evaluation of the restorations after a period of 10 months for the presence of secondary caries showed 
no statistically significant difference between the two groups.

\section{ACKNOWLEDG MENTS}

The authors would like to thank all the children, parents, schools and staff for their willing cooperation with the study.

\section{Manufacturers}

Papacarie: Formula one and A cao, B razil

Glass-ionomer cement: GC Gold label.

\section{REFERENCES}

1. B ussadori SK, M artins M D, Fernandes K P, Guedes CC, M otta LJ, RedaSH, Santos EM . Eval uation of in vitro biocompatibility of new product for chemical-mechanical caries removalPapacarie. Pesq Bras Odontoped Clin Integr Joao Pessoa 2005:5(3):253-59.

2. Michelle M ikhael A mmari, Luis Flavio M artins Moliterno. Chemo-mechanical caries removal: Current evidences. RBO 2005;62:125-27.
3. Irena Balčiunienǵ, Rūta Sabalaitǵ, Inga J uškienǵ. Chemomechanical caries removal for children. Stomatologija, B altic Dental and M axillofacial J ournal 2005;7:40-44.

4. Kotb RM, A bdella AA, EI K ateb M A, A hmed AM. Clinical evaluation of Papacarie in primary teeth. J Clin Pediatr Dent 2009;34(2):117-23.

5. Blake Bulloch, M ilton Tenenbein. V alidation of 2 pain scales for use in the pediatric emergency department. Pediatrics 2002;110(3):110-23

6. W ang J, W ang HM . Effects of three caries removal methods on children's dental fear evaluated by physiological measure. Shanghai K ou Qiang Y i Xue 2007;16(2):149-52.

7. Markovic D. Clinical evaluation of glass-ionomer tunnel restorations in primary molars: 36 months results. Australian Dental J ournal 2008;53:41-45.

8. B ussadori SK, Guedes CC, Hermida B runo M L, R am D. Chemomechanical removal of caries in an adolescent patient using a papain gel: Case report. J Clin Pediatr Dent Spring 2008; 32(3):177-80.

9. K irzioglu Z, Gurbuz T, Y ilmaz Y. Clinical evaluation of chemomechanical and mechanical caries removal: Status of the restorations at 3, 6, 9 and 12 months. Clin Oral Investig $M$ ar 2007;11(1):69-76. 